\title{
PRODUCCIÓN DE DISCURSOS SOBRE SEXUALIDAD \\ EN LA ESCUELA Y HETERONORMATIVIDAD: \\ EL CASO DE UN PROFESOR DE BIOLOGÍA
}

\author{
Production of discourses on sexuality \\ in schools and heteronormativity: \\ The case of a Biology teacher
}

\section{LAURA RANGEL BERNAL*}

\section{RESUMEN}

Esta investigación tuvo por objetivo estudiar el discurso sobre sexualidad en el caso de un profesor de Biología del nivel de educación secundaria. El estudio de caso sirvió como metodología, así como el análisis de entrevistas y las observaciones de clase, con herramientas del análisis crítico del discurso. La originalidad de la investigación radica en que aborda los discursos sobre sexualidad en las escuelas del nivel básico, los cuales, en México, están establecidos en los lineamientos curriculares, auque son los docentes quienes trasladan estos contenidos a las aulas y producen discursos en interacción con sus alumnos, de ahí la importancia de indagar cómo lo hacen y de qué recursos se valen. El principal hallazgo es que en el discurso convergen diversos enfoques sobre la sexualidad que tienden a naturalizar la heterosexualidad normativa, sus esquemas e instituciones. Además, el discurso del profesor enfatiza el carácter moral de las formas de regulación social de la sexualidad adolescente, mismas formas que restringen el ejercicio de esta última y obstaculizan el reconocimiento de dicho grupo poblacional como sujeto de derechos sexuales. Las limitaciones derivan de la metodología empleada, ya que para que los resultados puedan conducir a una generalización teórica se requiere un estudio de casos múltiples, el cual no pudo realizarse. Como conclusión, se discuten algunas implicaciones de lo anterior y se plantean futuras líneas de investigación.

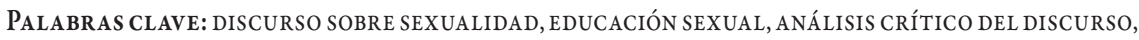
HETEROSEXUALIDAD NORMATIVA.

\footnotetext{
* Universidad Autónoma de Aguascalientes, Centro de Ciencias Sociales y Humanidades, Doctorado en Estudios Socioculturales. Correo electrónico: laura.rangelb@gmail.com.
} 


\section{Abstract}

This research was aimed at studying the discourse on sexuality, a professor of biology at the secondary level. The methodology used was the case study and analysis of interviews and classroom observations with tools of the approach of Critical Discourse Analysis. The originality of the research lies in the fact that it addresses the discourses on sexuality in the schools of basic education, which in Mexico, are set by the curriculum guidelines even though they are the teachers who move these contents to the classroom and produce speeches in interaction with their students, hence the importance of investigating how do and what resources they use. The main finding is that in the discourse converge diverse approaches to sexuality which tend to naturalize heterosexuality regulations, frameworks and institutions. In addition, the speech of the teacher emphasizes the moral character of the forms of social regulation imposed on adolescent sexuality, which restrict the exercise of the latter and hamper the recognition of this population group as a subject of sexual rights. The limitations derived from the methodology used, so that the results can lead to a theoretical generalization, it is necessary to make a multiple case study, which for various reasons could not be carried out. As a conclusion, it was discussed the implications of the findings and future lines of research.

KEYWORDS: DISCOURSE ON SEXUALITY, SEX EDUCATION, CRITICAL DISCOURSE ANALYSIS, NORMATIVE HETEROSEXUALITY.

Recepción: 23 de marzo de 2016.

Dictamen 1: 1 de junio de 2016.

Dictamen 2: 14 de junio de 2016. 
En México, los discursos sobre sexualidad que predominan en instituciones públicas (entre ellas, las educativas) se producen en el marco de un modelo de sexualidad fundado en los principios de la heterosexualidad normativa, modelo que invisibiliza y excluye otras sexualidades, pues limita a una sola las formas de concebir y ejercer la sexualidad. Lo anterior tiene repercusiones sociales, no solo en los contextos local y nacional, sino también en el global. En los últimos años, desde diversos frentes, se han dado avances en pro del reconocimiento de los derechos, la presencia social y la participación política de grupos de individuos que debido a su orientación e identidad sexual históricamente han sido objeto de marginación y discriminación. ${ }^{1}$ Estos avances reflejan cambios en la regulación institucional y estatal de la sexualidad que, a su vez, inciden en el orden sociosexual vigente.

Dado que la escuela no puede quedarse al margen de estos cambios, es necesario revisar los planteamientos a partir de los cuales se enseñan en ella los temas de sexualidad. Se requiere transformar el discurso de la educación sexual y, con ello, la educación sexual misma, lo cual no es tarea fácil debido a que ello implica desafiar, no solo los discursos dominantes, sino también la moral pública y sus defensores, por ello se requieren estudios que aborden esta problemática y propongan posibles vías de acción.

En el caso de la educación básica, ${ }^{2}$ los lineamientos curriculares establecen cómo y bajo qué enfoques se debe abordar la sexualidad en las escuelas; sin embargo, son los docentes quienes trasladan estos contenidos a las aulas y a partir de ellos producen discursos en interacción con sus alumnos. Para transformar tales discursos de modo que incluyan diversas perspectivas sobre la sexualidad, entre ellas aquellas que han sido excluidas sistemáticamente, es necesario conocer en profundidad los procesos de producción discursiva que tienen lugar en las aulas, a fin de determinar de qué manera estos discursos reproducen o desafían la ideología del discurso oficial sobre sexualidad, un tema que ha sido escasamente investigado en México.

Con base en los planteamientos anteriores, en la investigación de la cual deriva este artículo se analizó de manera cualitativa el discurso sobre sexualidad de un

\footnotetext{
1 Un ejemplo de los avances en materia jurídica en México es la determinación de la Suprema Corte de Justicia de la Nación que en 2015 declaró inconstitucionales los códigos civiles estatales que definen el matrimonio como la unión entre un hombre y una mujer y cuya finalidad es la procreación, bajo el argumento de que esta definición es discriminatoria porque impide el acceso de las parejas homosexuales a la institución del matrimonio (Semanario Judicial de la Nación, 2015).

2 En México, la educación básica comprende los niveles de preescolar, primaria y secundaria, a los que asiste la población infantil y adolescente entre los tres y quince años de edad. Desde 2011, con la publicación del Acuerdo 592 (Secretaría de Educación Pública, 2011), los planes y programas de estudio de estos tres niveles están articulados bajo los mismos estándares curriculares y principios pedagógicos, por lo que se le considera un currículum unificado.
} 
profesor de Biología con el objeto de dilucidar la construcción de tal discurso en el contexto de una clase dirigida a alumnos del primer grado de secundaria. Esto en el entendido de que el análisis de los procesos de producción discursiva de agentes específicos sirve, no solo para explicar cómo ocurren estos procesos en un contexto institucional (nivel micro), sino también contribuye a comprender las formas en las que operan las ideologías mediante el discurso para regular y normalizar el comportamiento sexual de la población (nivel macro).

Se empleó el estudio de caso instrumental como estrategia de gestión de conocimiento. Los datos fueron obtenidos por medio de la entrevista a un profesor que labora en una secundaria pública de la ciudad de Aguascalientes (en el estado de Aguascalientes, México), y a través de la grabación y el registro por escrito del total de sesiones de clase comprendidas en el periodo en que el docente impartió el tema de la sexualidad humana a dos grupos del primer grado en el ciclo lectivo 2014-2015. El estudio contó con un marco teórico-conceptual basado en los aportes del enfoque del análisis crítico del discurso.

El artículo está divido en cinco partes. En la primera de ellas se expone un panorama general de la conformación del discurso sobre sexualidad en la educación básica en México y se desarrollan de modo sucinto algunos argumentos para su problematización. En la segunda se explican los métodos y las técnicas empleados y se detalla el proceso de análisis de los datos. Los siguientes dos apartados están dedicados a la presentación y discusión de los hallazgos. En el apartado final se enuncian las conclusiones, se discuten las limitaciones del estudio y se plantean futuras líneas de investigación.

\section{EL DISCURSO SOBRE SEXUALIDAD EN LA EDUCACIÓN BÁSICA DE MÉXICO}

Dadas las controversias que suelen generarse a su alrededor, hablar de discursos sobre sexualidad en el ámbito educativo, en particular en la educación básica, implica aludir a contiendas libradas por diversos medios entre grupos que se disputan el derecho a decidir, no solo qué se enseña, sino también qué se dice sobre la sexualidad y cómo.

En México, han sido numerosos los momentos (sobre todo durante el siglo XX) en los que se ha debatido públicamente la pertinencia pedagógica y la relevancia social de ciertos temas relacionados con la sexualidad y, por ende, se ha discutido 
la necesidad de incluirlos en los planes de estudio, pero con especial énfasis se ha discutido su moralidad.

Pese a la frecuente oposición de un sector conservador conformado por organizaciones civiles y de padres de familia, en su mayoría de afiliación católica (véase Del Castillo, 2000, y Camacho Sandoval, 2008), con cada una de la reformas educativas efectuadas desde principios de los setenta hasta las primeras décadas del siglo XXI, se ha logrado integrar al currículo de la educación básica un conjunto de temas relativos a la sexualidad humana. Estos contenidos están incluidos en los planes de estudio de primaria y secundaria en el campo formativo "Exploración y conocimiento del mundo natural y social", bajo el rótulo "Salud sexual y reproductiva". ${ }^{3}$ Entre ellos figuran temas como las potencialidades de la sexualidad, la reproducción, la anticoncepción, la prevención de enfermedades de transmisión sexual y del embarazo en adolescentes (Secretaría de Educación Pública, 2011).

Con la integración de estos temas y de los enfoques pedagógicos bajo los cuales se enseñan, se ha constituido un modelo de educación sexual que ha dado pasos importantes en lo que respecta a la apertura para hablar de sexualidad con niños $y$ adolescentes en el espacio educativo. Sin embargo, este mantiene como base un modelo de sexualidad cuyos fundamentos ideológicos y esquemas coinciden con los de la normatividad heterosexual.

La normatividad heterosexual o heteronormatividad es una ideología que se ha constituido a lo largo de los siglos como el régimen social, cultural y político que condiciona de manera ubicua nuestro entendimiento y agencia con respecto del sexo y la sexualidad. El proceso mediante el cual se ha constituido como tal comprende la naturalizacion e institucionalización de esquemas de racionalización sobre la diferencia sexual y el género (Butler, 2001); de narrativas sobre el cuerpo, el nacimiento, el matrimonio, la familia y la muerte (Halberstam en Driver, 2008, p. 27); de códigos que regulan las prácticas sexuales y el acceso a parejas, a la vez que promueven la formación de sistemas de parentesco y herencia (cfr. Ross y Rapp, 1997), y de jerarquías de poder en las cuales las mujeres, lo femenino y lo homosexual guardan una relación de subordinación con respecto de los hombres, lo masculino y lo heterosexual (véase Richardson, 2000).

\footnotetext{
3 El término "salud sexual" ha sido criticado por Amuchástegui (2006) debido a que se trata de una expresión polisémica cuyo significado e implicaciones no siempre se definen con claridad en los discursos. Dada su ambigüedad y valoración positiva que se le atribuye, grupos con posturas ideológicas contrarias se lo han apropiado y lo usan con fines políticos opuestos (p. 209).
} 
La heteronormatividad, como otras ideologías, opera a través del discurso para establecerse como hegemónica. Procede por medio tanto de la esencialización de las identidades sexuales y genéricas como de la normalización de la cultura y del deseo heterosexuales (cfr. Bhatacharyya, 2002), entendido, este último, como fundador de un supuesto orden natural universal (Granados Cosme, 2006). En el ámbito escolar, esta ideología promueve el establecimiento de mecanismos de normalización de las identidades sexuales dispuestos para constituir a los sujetos de la educación como sujetos heterosexuales (Flores, 2008; Theumer y García Muñoz, 2011).

A fin de indagar y explicar las formas en que se reproduce la ideología heteronormativa en el discurso educativo, la investigación se basó en planteamientos teórico-metodológicos del enfoque del análisis crítico del discurso (ACD). Este enfoque propone la existencia de una relación dialéctica entre producción discursiva, reproducción ideológica y el mantenimiento de relaciones desiguales de poder y de las estructuras de dominación actuales.

Como lo expresa Teun van Dijk, "las ideologías fundamentales de nuestra sociedad están basadas en prácticas discursivas difundidas" (2004, p. 18). Ello se debe a las cualidades y facultades propias del lenguaje que facilitan la difusión de contenidos ideológicos a través de la producción discursiva. En función de esta relación dialéctica, en el ACD se conjugan dos perspectivas: la social y la lingüística. Ambas permiten el estudio crítico del discurso de las instituciones y de los diversos actores sociales, el análisis de los usos y funciones sociales del lenguaje en contexto y la explicación del modo en que este contribuye a configurar el orden social vigente.

En este sentido, se planteó analizar el discurso sobre sexualidad de actores educativos, en el entendido que este tipo de discursos contribuye a sostener la hegemonía de la heteronormatividad a través de la educación formal y porque se afirma que en la transformación discursiva yace una de las claves para contrarrestar dicha hegemonía.

\section{Metodología}

Con la finalidad de obtener los datos necesarios para la investigación, se realizó un estudio de caso. Con los datos obtenidos se conformó un corpus que fue analizado cualitativamente mediante el uso de herramientas metodológicas y analíticas tomadas del enfoque lingüístico del análisis crítico del discurso, más específicamente del modelo de análisis histórico discursivo de Reisigl y Wodak (2008). 
Se eligió el estudio de caso porque este método es apropiado para contestar preguntas sobre cómo y por qué ocurren ciertos fenómenos sociales (Yin, 1984, p. 29), es decir, porque alude a procesos y a explicaciones holísticas de los mismos fenómenos y porque, como lo explica Sayago (2012), el uso instrumental del estudio de caso permite analizar representaciones discursivas de forma cualitativa.

Puesto que la investigación se centró en el discurso sobre sexualidad dirigido a adolescentes, se decidió realizar el trabajo de campo en escuelas del nivel secundaria. Cifras de la Secretaría de Educación Pública (2015) indican que cerca de 90 por ciento de los adolescentes que se encuentran entre los 12 y 14 años de edad cursan la educación secundaria en México. Este elevado porcentaje devela el alcance que tienen los discursos que se producen en dicho nivel educativo entre la población adolescente, lo cual contó como un aspecto más para determinar la relevancia del estudio. El profesor participante se seleccionó mediante muestreo por conveniencia, tras la aplicación de un cuestionario exploratorio en nueve secundarias públicas de la ciudad de Aguascalientes.

Se diseñó una entrevista semiestructurada cuyas preguntas se orientaron hacia la indagación de la forma en que el maestro abordaba el tema de la sexualidad en sus clases de Biología con los dos grupos del primer grado del turno vespertino que en ese entonces estaban a su cargo. Tras la entrevista, se realizaron observaciones de las clases durante el lapso en que el maestro impartió el tema de la sexualidad humana (aproximadamente un mes) en el ciclo lectivo 2014-2015. Las trece sesiones de clases a las que asistió la investigadora en calidad de observadora no participante fueron grabadas en audio y, posteriormente, transcritas. De forma paralela a la grabación de las clases, se registró por escrito lo acontecido en cada sesión.

Los datos fueron analizados en dos momentos. En el primero de ellos se aplicó el método de la comparación constante (Osses Bustingorry, Sánchez Tapia e Ibáñez Mansilla, 2006) para codificar los datos. Después, mediante la elaboración de tablas analíticas en las que se agruparon los códigos de acuerdo con las afinidades identificadas entre ellos, se construyeron las categorías y subcategorías. Estas tienen fundamento empírico, pero también se basan en conceptos localizados en la revisión de la literatura. Siguiendo el modelo analítico de Van Reisigl y Wodak (2008), esta primera fase del análisis incluyó también la identificación de los tópicos, o sea, de los temas centrales que aparecen en el discurso analizado. Asimismo se identificaron los diferentes discursos que lo componen, lo que a su vez permitió dilucidar su configuración, es decir, saber qué discursos lo alimentan, de qué enfoques sobre la sexualidad provienen estos discursos y conocer cuál es la relación 


\section{entre ellos. En este artículo se presentan únicamente los resultados de esta primera fase del análisis, es decir, el análisis categorial.}

\section{RESUlTADOS}

\section{En el cuadro 1 se presenta el total de las categorías con sus respectivas definiciones.}

\section{Cuadro 1. Categorías y subCATEgorías}

1 Sexualidad: conjunto de creencias, prácticas, comportamientos, símbolos y otros elementos culturales asociados con la sexualidad y con el uso cotidiano de los cuerpos sexuados.
Subcategoría 1.1 Potencialidades de la sexualidad: posibles manifestaciones del ejercicio y vivencia de la sexualidad, entre las que figuran la afectividad, el erotismo, el género y la reproduc- tividad.

2 Género: conjunto de ideas, creencias y representaciones culturales sobre el género. Incluye los roles, identidades, estereotipos y símbolos asociados con cada género.

\begin{tabular}{ll}
\hline Subcategoría 2.1 & $\begin{array}{l}\text { Concepto de género: elementos teórico-conceptuales a través de los cuales se define } \\
\text { el género en el discurso. }\end{array}$ \\
\hline Subcategoría 2.2 & $\begin{array}{l}\text { Estereotipos y roles de género: conjunto de representaciones estereotípicas de lo } \\
\text { masculino y lo femenino y concepciones relativas a la división sexual del trabajo. }\end{array}$
\end{tabular}

3 Información sobre sexualidad: ideas sobre la producción, difusión y uso de información relacionada con el sexo y la sexualidad que privilegian el conocimiento científico sobre otros tipos de conocimiento.

4 Modelos de pareja y familia: concepciones y representaciones de la pareja heterosexual y la familia nuclear. Implican la práctica de la fidelidad, de la monogamia vitalicia, entre otras.

5 Dimensión axiológica y moral de la sexualidad: conjunto de valores y disposiciones morales que norman y regulan la sexualidad y su ejercicio, en el que la responsabilidad actúa como valor rector.

6 Regulación social de la sexualidad adolescente: indicios de la acción normativa que instituciones como la escuela, la familia y la Iglesia, junto con otras entidades sociales, ejercen sobre el comportamiento, las prácticas sexuales y el uso de los cuerpos de los adolescentes.

7 Discurso construccionista de la sexualidad: alude a una concepción de la sexualidad entendida como construcción social e históricamente determinada, atravesada por relaciones de poder (Ojeda Sánchez, cit. en Parrini, 2007)

8 Discurso biologicista de la sexualidad: provee explicaciones sobre el sexo y la sexualidad basándose en el modelo de producción de conocimiento propio de la biología.

9 Discurso sobre equidad de género: habla de diferencias o desigualdades sociales, culturales y económicas que históricamente han prevalecido entre hombres y mujeres. Promueve la equidad, la transformación de roles tradicionales y la eliminación de estereotipos de género.

10 Discurso del enfoque biomédico: trata de la prevención de enfermedades transmitidas por contacto genital y del embarazo en adolescentes, ambos considerados riesgos inherentes al ejercicio temprano de la sexualidad. Por ello advierte de la necesidad de evitarlos o prevenirlos. 
11 Discurso sobre la planeación familiar: recomienda planear con anticipación, de manera consciente y deliberada, la ocurrencia de embarazos con base en el conocimiento del uso y funcionamiento de métodos anticonceptivos. También plantea que ciertas circunstancias de vida son mejores o más apropiadas para tener hijos que otras.

12 Discurso sobre la adolescencia: se basa en un modelo de desarrollo humano en el que la edad adulta es considerada la etapa cumbre, al tiempo que concibe a los adolescentes como seres en desarrollo que carecen de o que aún no han adquirido plenamente las capacidades y habilidades necesarias para participar como agentes independientes en una sociedad dirigida por adultos.

Fuente: Elaboración propia.

\section{Discusión}

Para interpretar las categorías y comprender la relación entre ellas, resulta conveniente dividirlas en dos grupos. Las primeras seis (véase el cuadro 1) corresponden a unidades de sentido identificadas en el discurso, mientras que las seis restantes, a los discursos de los cuales se alimenta el discurso analizado.

Las categorías 1 y 2 , junto con sus respectivas subcategorías, y, en cierta medida, la categoría 3 , tienen correspondencia con los contenidos que el programa de la asignatura marca para la primera parte del bloque cuatro: "La reproducción y la continuidad de la vida” (Secretaría de Educación Pública, 2013, p. 45). En cuanto a las categorías 7 a la 12 (véase el cuadro 1), estas corresponden a los discursos que alimentan el discurso analizado. Tales discursos provienen de diversos enfoques sobre la sexualidad que han sido institucionalizados con cada una de las reformas hechas al currículum de la educación básica en los últimos cincuenta años. ${ }^{4}$ En el discurso del profesor, la aparición de estos discursos con sus respectivos enfoques es consistente con lo que plantea el discurso curricular (cfr. Secretaría de Educación Pública, 2011 y 2013).

En contraste, las categorías 4, modelo depareja y familia; 5, dimensión axiológicamoral de la sexualidad, y 6 , regulación social de la sexualidad adolescente, no tienen correspondencia directa con el discurso curricular, lo cual indica que los elementos que las componen fueron traídos a cuenta por el mismo profesor. Esto último es relevante porque es muestra de la agencia del docente en la producción discursiva,

${ }^{4}$ Entre estos enfoques se encuentran el biologicista, el demográfico, el biomédico, el epidemiológico, el construccionista y el de género. Se sugiere consultar Morgade y Alonso (2008) para saber más sobre los enfoques bajo los cuales se ha enseñado la educación sexual en Latinoamérica. 
toda vez que él decidió integrar elementos que no están planteados explícitamente en el programa de la asignatura a fin de construir y dar coherencia a su propio discurso.

La relevancia de esta acción reside también en el hecho de que mediante la integración de nociones y de referencias de situaciones, prácticas, comportamientos, tipos de relaciones interpersonales, normas sociales, entre otros, cuyo rasgo común es su naturaleza moral, el profesor codifica contenidos ideológicos asociados con la moral conservadora católica, que refuerzan el carácter heteronormativo de su discurso. Por medio del análisis de fragmentos seleccionados del corpus que se presentan a continuación puede verse de qué modo opera esto en el discurso.

\section{LA SEXUALIDAD DEFINIDA SEGÚN LA NORMA HETEROSEXUAL}

En primer lugar, tenemos que el profesor ubica la sexualidad y su ejercicio en un contexto heterosexual y adulto desde la primera sesión de clase como puede verse en el siguiente fragmento.

(1)

Maestro: ¿Qué es la atracción sexual?

Fredy: Es cuando una hembra o un macho o un... un masculino, o un... ¡cómo se llama?, una femenina usa feromonas para atraer al macho o a la hembra... ¿Sí?

Maestro: Algo así.

Fredy: Cuando una mujer es, este... acásensual; también un hombre que ya conquistó a la vieja. Maestro: Por eso, ¿qué es? Cuando ustedes entran a la secundaria van reteniendo su atracción sexual [...] ¿sí? ¿Por qué la van deteniendo? Es algo parecido a la atracción sexual, [es] atraer... Fredy: A la mujer o al hombre.

Maestro: Al sexo opuesto.

Brandon: La atracción del sexo opuesto.

Maestro: Atracción al sexo opuesto: hombre, mujer; mujer, hombre [...]

Cabe señalar que tanto en el discurso del profesor como en el del currículum oficial, la noción de orientación sexual está ausente. ${ }^{5}$ Asimismo, en una revisión del Acuerdo

5 Este parece ser un rasgo común de los programas de educación sexual de diversos países, entre los que se encuentran Chiley Canadá(Martínez,Menesesy Sarabia, 2004). Atribuyo la ausencia de esta noción a una estrategia discursiva dispuesta por los diseñadores del currículum para mantener la apariencia de neutralidad de este último con respecto de los temas de la homosexualidad y la diversidad sexual, además de constituir una evidencia de heterosexismo. 
592 (Secretaría de Educación Pública, 2011), un documento de 640 páginas que trata sobre la articulación curricular de los tres niveles de la educación básica y que comprende los planes y programas de preescolar, primaria y secundaria, no se encontró una sola incidencia de vocablos relacionados con esta noción. ${ }^{6}$ Sin embargo, toda vez que la sexualidad se inserta en el bloque temático de la reproducción y al hacer referencia a prácticas sexuales directamente vinculadas con la reproducción (como es el coito vaginal), se entiende que el discurso está refiriéndose únicamente a prácticas y relaciones de tipo heterosexual, lo cual también se advierte en el siguiente fragmento.

Fredy: Profe, ¿la sexualidad es cuando tienen relaciones sexuales?

Maestro: [para contestar, lee de nuevo un fragmento del libro de texto que uno de los alumnos ya había leído previamente] "En cambio, la sexualidad es más amplia, tiene relación con su forma de pensar de ser, de relacionarse”. Lo que tú me estás diciendo es el acto sexual, es el coito, es la... es la unión de dos sextos, sexos, perdón. Es la unión de dos sexos. Esa es la, ¿sí?, la relación sexual [...]

Nótese que en el fragmento (1), frente a la pregunta qué es la atracción sexual, Fredy, uno de los alumnos, deja abierta, hasta cierto punto, la opción al responder "al macho o a la hembra" y "al hombre o a la mujer". En su siguiente intervención, el maestro cancela la ambigüedad de la construcción disyuntiva planteada en las repuestas del alumno al especificar que la atracción sexual es la "atracción al sexo opuesto", y plantea dos combinaciones — hombre-mujer, mujer-hombre-, que finalmente resultan ser la misma, pero que es necesario mencionar, pues tienen el efecto de suprimir la posibilidad de considerar otras combinaciones (hombrehombre, mujer-mujer) que estarían refiriéndose a tipos de atracción, prácticas sexuales e identidades que se encuentran al margen de los modelos de sexualidad fundados en la heteronormatividad.

Su definición de relaciones sexuales, acto sexual o coito, que aparece en el fragmento (2) como "la unión de dos sexos", refuerza esta concepción, con lo que otras sexualidades y otras prácticas sexuales no reconocidas por la norma heterosexual quedan fuera del discurso.

\footnotetext{
6 Se buscaron los vocablos orientación sexual, heterosexualidad, homosexualidad y otros derivados. Tampoco se encontraron referencias a la diversidad sexual, a pesar de que, según el Acuerdo 592 (Secretaría de Educación Pública, 2011), uno de los principios pedagógicos que rigen al currículum es "favorecer la inclusión para atender a la diversidad" (p. 27).
} 


\section{REGULACIÓN SOCIAL DE LA SEXUALIDAD ADOLESCENTE}

Por otra parte, en una de sus intervenciones en el fragmento (1), el maestro menciona que cuando los alumnos ingresan al nivel secundaria "van reteniendo su atracción", con lo cual él hace referencia a la facultad que la escuela, como institución social, tiene para regular la sexualidad de los adolescentes. Un ejemplo adicional lo proporciona el siguiente fragmento.

(3)

Maestro: Por eso las muchachas se retienen mucho, porque en la secundaria se prohíbe mucho lo que es lápiz labial, la pintadita de ojos, el moñito, el aretito, lo que a ellas les hacen sentir tranquilas, a gusto. La blusita ombliguera que cuando van a la primaria la llevan, ¿sí o no?, la falda dos cuartas arriba de la rodilla, cuando salen de la primaria [...]

El tema de la regulación social de la sexualidad no está incluido en el currículum escolar, al menos no de manera explícita. ${ }^{7}$ No obstante, el maestro habla con relativa frecuencia de los mecanismos e instrumentos a través de los cuales se regula la sexualidad de los adolescentes en los planteles educativos. En este sentido refiere la existencia de reglamentos que prohíben y sancionan determinadas conductas por ser consideradas manifestaciones impropias de la sexualidad (en particular de la femenina) en el entorno escolar. Al respecto, en el fragmento (3) menciona el uso de maquillaje, de ciertas prendas de vestir y accesorios, pues se les asocia culturalmente con el ejercicio de la sexualidad femenina con fines de seducción, lo cual es inadmisible en una institución supuestamente neutral o asexual (véase Duvet, 2007). Es importante señalar que la contraparte, esto es, las manifestaciones de la sexualidad masculina, no es abordada por el profesor, por ello puede hablarse de una distinción fundamentada en nociones de género que percibe la sexualidad femenina como disruptiva y la masculina como neutral.

Además de la escuela, el maestro reconoce a la Iglesia y a la familia como instituciones reguladoras de la sexualidad adolescente; la Iglesia, a través del sacramento del matrimonio y de la moral católica, que él parece reconocer como ubicua en el contexto en que desempeña su labor docente; la familia, como institución (en palabras del profesor) "formativa y normativa que enseña normas sociales, sexuales,

7 Aunque se entiende que la educación sexual impartida por el Estado es un dispositivo que regula la sexualidad de la población a través de la educación formal (cfr. Foucault, 1978, y Jäger, 2003). 
de amistad y compañerismo para que los hijos [entiéndase los adolescentes] sepan cómo desenvolverse en la sociedad".

Asimismo, el maestro habla repetidamente de la importancia del noviazgo como espacio para la comunicación y el conocimiento del otro previo al matrimonio, por ello, dadas las funciones que le atribuye, el noviazgo puede interpretarse como una instancia reguladora más de la sexualidad adolescente. El fragmento que sigue ilustra este punto.

(4)

Maestro: [...] Tengan mucho cuidado, para eso es el noviazgo, no se les niega que tengan novio, pero es para que se conozcan, para que se agraden, no para andar perreando ${ }^{8}$ en los bailes [...] Aquíla situación es, con ustedes, que entiendan la confianza que se debe adquirir en el noviazgo [...]

El noviazgo figura como una subinstitución del matrimonio en la que los adolescentes pueden participar, aunque no exactamente para ejercer su sexualidad o, dado el caso, pueden hacerlo pero de forma muy restringida, pues se explica que su función no es esa, sino la de establecer las presupuestas bases de un matrimonio exitoso y duradero. Por ello se resaltan aspectos como la comunicación, la confianza, el entendimiento mutuo, el afecto, etcétera.

Hablar de las formas en las que se regula la sexualidad en este discurso implica reconocer que, más allá de lo planteado por el modelo de educación sexual del currículum escolar, el cual está más bien orientado a promover la autorregulación con base en el conocimiento científico referente a la sexualidad que se adquiere en la escuela, existen fuerzas y mecanismos socioculturales que actúan de manera puntual para regular y normalizar el comportamiento sexual en función de lo establecido por las ideologías de sexualidad dominantes.

\section{Modelos DE PAREJA Y FAMILIA}

Otro aspecto que evidencia la codificación de una ideología de sexualidad particular en este discurso son los modelos de pareja y familia a los que se hace referencia, ambos basados en principios de la moral conservadora católica.

\footnotetext{
8 El perreo es un baile de origen puertorriqueño que en los últimos años ha generado controversia entre algunos grupos debido a que consiste en realizar movimientos considerados sexualmente explícitos.
} 
Para entender cómo funciona esto es necesario señalar que los documentos oficiales en los cuales los docentes deben basar la impartición de la materia de Ciencias I (el plan de estudios, el programa de la asignatura y la guía para el maestro) no señalan explícitamente modelos de pareja ni de familia al referirse al tema de la sexualidad. En este sentido, el discurso curricular no ubica el ejercicio de la sexualidad en un lugar definido, ${ }^{9}$ pero el profesor sílo hace, toda vez que para hablar de sexualidad se requiere aludir a prácticas sexuales y sociales, tipos de relaciones interpersonales, sistemas de creencias y valores, así como a los modelos culturales que articulan todos estos elementos, y para ello es necesario aterrizarlos, por así decirlo, en algún punto. De este modo, el profesor centra el ejercicio de la sexualidad en lo que podemos denominar aquí (debido a la interdependencia existente entre estas dos instituciones) la unidad matrimonio-familia.

Son varios los efectos que tiene esto en el discurso. En primer lugar, ubicar el ejercicio de la sexualidad en la unidad matrimonio-familia normaliza la heterosexualidad, como se explica en un subapartado anterior. En segundo lugar, ciñe el ejercicio de la sexualidad a la edad adulta, cuando es deseable actualmente que las personas se casen y formen familias, dejando de lado el planteamiento de la sexualidad adolescente con las características y problemáticas que le son propias.

En tercer lugar, se impone y reproduce un patrón cognitivo-discursivo que iguala la sexualidad con la procreación, pese a que en una de las primeras sesiones de clase, y de acuerdo con lo establecido por el programa de la asignatura, el profesor trata de asociar la reproducción con la reproductividad, es decir, presentarla como una potencialidad más de la sexualidad, y no como un aspecto invariablemente ligado a ella o como un efecto inevitable de su ejercicio. El que a fin de cuentas se imponga la noción de sexualidad igual a procreación impide hablar, entre otras cosas, de maternidad y paternidad voluntarias.

En cuarto lugar, se naturaliza una narrativa que concibe la vida humana como una sucesión de acontecimientos cuyos hitos están marcados por eventos vinculados con la experiencia heterosexual social y culturalmente normada, como son tener una pareja, casarse, tener hijos, etcétera. Esta narrativa puede considerarse como parte de lo que Bhattacharyya (2002) denomina la cultura heterosexual, la cual tiene la función de legitimar y perpetuar la ideología heteronormativa.

\footnotetext{
9 Lo cual, probablemente, sea otro intento por mantener la apariencia de neutralidad ideológica del currículum escolar; aunque es clara su postura, toda vez que el tema de la sexualidad en el área de las Ciencias Naturales no constituye una unidad temática en sí misma, sino que se inserta en el bloque de la reproducción, lo cual mantiene un vínculo, que puede ser puesto en entredicho, entre sexualidad y procreación.
} 


\section{MORAL RELIGIOSA Y DISCURSOS HETERONORMATIVOS}

Un último efecto de ubicar el ejercicio (cabe señalar aquí, legítimo y seguro) de la sexualidad en los confines de la familia nuclear y del matrimonio heterosexual es que se la domestica (o, en palabras de Foucault, se la disciplina), en este caso, a través de la introducción de nociones morales ligadas con la ideología sexual religiosa como son la monogamia vitalicia y la fidelidad. Este punto se ilustra en el fragmento que se muestra a continuación. En una de las sesiones de clase en las que se abordó el tema de las enfermedades de transmisión sexual, el maestro pregunta quién transmite el virus del papiloma humano. Él mismo contesta:

\section{(5)}

Maestro: Los hombres por medio de relación sexual [lo anota en el pizarrón]. Por medio de relaciones sexuales. Aquí la idea es de que se orienten, no de que lo pongan en práctica, pero que se orienten; de que cuando ya que tengan su pareja definitiva, pues que sea eso: una pareja definitiva, en la que va a haber respeto, cordialidad, amistad, compañerismo y, sobre todo, comunicación. Si en la pareja no hay comunicación, no nos vamos a entender, ¿sí? Va a haber algunas personas que acepten lo que viene siendo la infidelidad, otras no. Entons [sic] mientras, este, el hombre participe en muchas relaciones sexuales con diferentes tipos de mujeres, va a haber alguna o va a haber varias que le peguen el bichito o que le peguen el virus en el pene y que luego a quien se lo transmitan va a ser a la mujer, por lo general viene siendo a su esposa $[\ldots]$

En el fragmento (5), el profesor integra a su discurso las nociones de fidelidad y monogamia vitalicia al hablar de tener una pareja definitiva. Ambas nociones son planteadas, no solo como normas morales de la conyugalidad, sino también como formas de prevenir y evitar el contagio de enfermedades de transmisión sexual.

Este punto hay que resaltarlo, ya que esta postura coincide con la de la moral católica que, según Amuchástegui (2006), "ha querido definir la 'salud sexual' a través del concepto de 'sexualidad sana', misma que estaría confinada al coito reproductivo dentro [del] matrimonio" (p. 212). Con base en este concepto, grupos conservadores de afiliación católica promueven la fidelidad como forma primordial de prevenir enfermedades de transmisión sexual, colocándola antes o, incluso, en lugar del uso de métodos de barrera como el condón masculino y femenino, que tienen un alto margen de efectividad en la prevención de este tipo de infecciones y que funcionan como anticonceptivos. 
La idea de que la sexualidad solo puede ejercerse legítimamente en los confines del matrimonio y que los posibles efectos de su ejercicio (el embarazo, principalmente) solo pueden sobrellevarse de forma digna si se está casado conlleva la recomendación de postergar el inicio de la actividad sexual a la edad adulta, cuando es socialmente aceptable y, en algunos casos, legal que las personas adolescentes se casen. Esta recomendación aparece de forma más bien velada en el discurso ${ }^{10}$ y se desarrolla a través de estrategias discursivas mediante las cuales se resaltan los efectos negativos del ejercicio de la sexualidad, no solo para sí mismo, sino también para otros, al tiempo que se minimizan o invisibilizan sus efectos positivos. Esto puede verse en el fragmento que sigue, en el cual el profesor discute las consecuencias del embarazo en la adolescencia.

\section{(6)}

Maestro: Así de sencillito, si nos quedamos embarazados tenemos muchos gastos; perdemos muchas libertades de adolescente, de jóvenes, esperando tener la principal responsabilidad de lo que viene siendo nuestro bebé o nuestro hijo, ¿̇í? Entonces, aquí nuestra tarea es empezar a hacer conciencia de que van a terminar su adolescencia. [Los embarazos en adolescentes] abarcan muchas, muchas condiciones, inclusive la agresión sexual, la violencia sexual, e inclusive la malformación de mi cuerpo o la malformación del producto o del bebé que vaya a tener, porque si mi cuerpo ahorita todavía no está bien definido, bien maduro, entonces voy a procrear una criatura que tenga diferentes tipos de problemas, ya sean corporales, mentales, psicológicos o simplemente de, del organismo, ¿`sí? [...]

La recomendación de aplazar la actividad sexual al matrimonio, que constituye una prescripción de abstinencia sexual impuesta a la población adolescente, es producto de la convergencia de varios discursos. Por un lado tenemos el discurso del enfoque biomédico que representa las prácticas sexuales durante la adolescencia como riesgosas debido a los posibles efectos negativos en la salud de las personas. En segundo lugar está el discurso de la moral religiosa ${ }^{11}$ que prohíbe de manera explícita toda actividad sexual previa al matrimonio. En tercer lugar está el discurso

10 Ejemplo de esto lo encontramos en el fragmento (5) donde dice: "aquí la idea es de que se orienten, no de que lo pongan en práctica, pero que se orienten", esto significa que la "orientación" provista por la educación sexual debe servir a los adolescentes para el ejercicio futuro de su sexualidad en pareja, mientras que las prácticas sexuales en la adolescencia quedan de cierto modo vedadas.

11 Cabe señalar que los principios morales que prohíben o sancionan la actividad sexual prematrimonial no son exclusivos del catolicismo, sino que son compartidos por las principales religiones del mundo como son las diferentes vertientes del cristianismo, el judaísmo, el islamismo, etcétera. 
sobre la adolescencia que representa a esta etapa como un periodo de crecimiento y desarrollo en la que los individuos no están ni física, ni mental, ni emocionalmente preparados para afrontar los "riesgos" de la sexualidad.

Por último, encontramos lo que Meneses, Martínez y Sarabia (2006) denominan discursos de la sexualidad como violencia y como victimización. Ambos hacen "énfasis en los abusos sexuales, la transmisión del sida y las enfermedades venéreas, en detrimento de otros factores como los métodos contraceptivos, los derechos sexuales y reproductivos, el consentimiento y la decisión informada" (2006, p. 4), al tiempo que hacen "hincapié en la vulnerabilidad de la mujer ante el deseo masculino" (2006, p. 4). Este último discurso se basa en concepciones esencialistas sobre la sexualidad que se entrelazan con el género, en tanto que se funda en las antinomias dominación/sumisión y actividad/pasividad, las cuales otorgan agencia a los hombres y lo masculino en lo que respecta al ejercicio de la sexualidad, pero niegan esa misma agencia a las mujeres y lo femenino.

La prescripción de abstinencia sexual que deviene de esta conjunción de discursos constituye, además, una forma de evitar lidiar de manera directa con la multiplicidad de manifestaciones, prácticas, significados y problemáticas propias de la sexualidad adolescente. Una de las repercusiones de esta evitación sistemática es la imposibilidad de que se reconozcan de modo pleno los derechos sexuales de los adolescentes, en tanto que no se les considera cabalmente como sujetos de tales derechos.

\section{Conclusiones}

El objetivo de la investigación consistió en analizar el discurso sobre sexualidad de un profesor de Biología del nivel secundaria para, a partir de ello, proponer explicaciones sobre cómo este tipo de discursos reproducen la ideología sexual predominante. Se encontró que este discurso coincide en varios puntos con lo que indica el currículum escolar en materia de educación sexual, puesto que se abordan todos los temas señalados en el mismo currículum y se mencionan elementos pertenecientes a los diferentes discursos y enfoques que conforman el modelo oficial de educación sexual del área de las Ciencias Naturales. Sin embargo, mientras que en el currículum predomina el discurso biomédico que resalta la importancia de contar con un conocimiento científico sobre asuntos relativos a la sexualidad para tomar decisiones informadas, prevenir embarazos y enfermedades de transmisión sexual (Secretaría 
de Educación Pública, 2013, pp. 39 y 40), en el discurso del profesor se habla más de una ética de la sexualidad fundada en una moral conservadora de carácter religioso.

De este modo, la sexualidad es presentada como altamente regulada por normas socioculturales apegadas a una moral particular. Esta representación incluye la naturalización de la heterosexualidad, sus esquemas, sus narrativas, su cultura y sus mecanismos de regulación, por lo que se habla de la sexualidad adulta, doméstica, monógama y reproductiva como el modelo ideal para su ejercicio o, al menos, como el único legítimo, seguro (en lo que respecta al cuidado de la salud) y socialmente aceptable.

El que el maestro reproduzca un contenido ideológico heteronormativo en su discurso podría explicarse por el contexto en que fue formado, por sus propias experiencias de vida, por las restricciones institucionales que le son impuestas en su labor docente, o por los discursos que circulan en diversos medios. Sin embargo, es necesario recordar que todos estos elementos condicionan, mas no tendrían que impedir, la posibilidad de que los agentes que participan en la producción de discursos sociales conciban la sexualidad de otras maneras, que consideren perspectivas más críticas, que reconozcan la diversidad de prácticas, significados y placeres, y vean el cuerpo, no solo como un organismo susceptible de contraer enfermedades o de reproducirse, sino también como un espacio de poder y resistencia. ¿De qué modo se podría conseguir esto? Una forma sería que los docentes reconocieran formas alternativas de concebir la sexualidad en sus clases, formas que no están centradas en la pareja heterosexual adulta, cuyo ejercicio no está restringido por la moral dominante y cuyo fin primordial no es la reproducción, sino el ejercicio del deseo y el placer.

En cuanto a las limitaciones de la investigación, un estudio de caso como el que se realizó permite conocer en profundidad la complejidad de los procesos de producción discursiva que constituyen el objeto primordial de los análisis microdiscursivos, por lo que existe la posibilidad de someterlos a una generalización teórica, es decir, considerarlos como base para la generación de teoría (Yin, 1984). Sin embargo, para llegar a tal punto habría sido necesario realizar procedimientos de replicación que exigirían la elaboración de un diseño de investigación de casos múltiples, lo cual, por diversas razones, no pudo ser realizado.

Por otra parte, este estudio ha permitido identificar futuras líneas de investigación relacionadas con la producción y reproducción de discursos sobre sexualidad que realizan agentes sociales en contextos institucionales específicos (ya sea educativos, académicos o del sector salud), así como con los procesos de generación y sedimentación de significados sobre la sexualidad y los efectos que dichos procesos tienen en la 
cognición social y en la generación de discursos institucionales. Como se mencionó anteriormente, la posibilidad de construir teoría a partir de un estudio de caso yace en la realización de investigaciones que continúen indagando en la línea analítica planteada, por lo que es importante persistir en la realización de tales estudios.

\section{BiBLIOGRAFÍA}

Amuchástegui, A. (2006). Procesos subjetivos de ciudadanía sexual en México: Heteronormatividad y salud. En F. C. Cáceres, G. Careaga, T. Frasca y M. Pecheny (eds.). Sexualidad, estigma y derechos humanos. Desafios para el acceso a la salud en América Latina (pp. 209-221). Lima, Perú: Universidad Peruana Cayetano Heredia.

Bhattacharyya, G. (2002). Sexuality and Society. An Introduction. Londres, Reino Unido: Routledge.

Butler, J. (2001). El género en disputa. El feminismo y la subversión de la identidad. Distrito Federal, México: Paidós, Universidad Nacional Autónoma de México, Programa Universitario de Estudios de Género.

Cáceres F. C.; Careaga, G.; Frasca, T., y Pecheny, M. (eds.) (2006). Sexualidad, estigma y derechos humanos. Desafios para el acceso a la salud en América Latina. Lima, Perú: Universidad Peruana Cayetano Heredia.

Camacho Sandoval, S. (2008). El fantasma del sexo en los libros de la SEP. Oposición a los libros de texto en Aguascalientes, 1930-2006. Parteaguas, 14(otoño): 26-31.

Del Castillo Troncoso, A. (2000). La polémica en torno a la educación sexual en la ciudad de México durante la década de los años treinta. Estudios Sociológicos, 1(XVIII): 203-226. Recuperado de http://www.redalyc.org/pdf/598/59805207.pdf.

Duvet, F. (2007). El declive y la mutación de la institución. Revisa de Antropología Social, 16: 36-66.

Driver, S. (ed.) (2008). Queer youth cultures. Nueva York, Estados Unidos: State University of New York Press.

FLores, V. (2008). Entre secretos y silencios. La ignorancia como política de conocimiento y práctica de (hetero)normalización. Revista Trabajo Social, 18: 14-21. Recuperado de http://www.revistas.unam.mx/index.php/ents/article/view/19514.

Foucault,M.(1978).Verdadypoder.EntrevistaconM.Fontana.L'arc,70: 16-26.Recuperado de http://www.ram-wan.net/restrepo/poder/verdad\%20y\%20poder.pdf. 
Granados Cosme, J. A. (2002). Orden sexual y alteridad. La homofobia masculina en el espejo. Nueva Antropologia, 61(XVIII): 79-97. Recuperado de http://www. redalyc.org/articulo.oa?id=15906104.

Halberstam, S. (2008). What's that Smell: Queer Temporalities and Subcultural Lives. En S. Driver (ed.). Queer Youth Cultures (pp. 27-50). Nueva York, Estados Unidos: State University of New York Press.

Jäger, S. (2001). Discourse and Knowledge: Theoretical and Methodological Aspects of Critical Discourse and Dispositive Analysis. En R. Wodak y M. Meyer (eds.). Methods of Critical Discourse Analysis (pp. 32-62). Londres, Gran Bretaña: Sage Publications.

Lancaster, R., y Di Leonardo, M. (eds.) (1997). The Gender/Sexuality Reader. Culture, History, Political Economy. Nueva York, Estados Unidos: Routledge.

Martínez, A.; Meneses, A., y Sarabia, D. (2004). La educación para la salud sexual de los adolescentes en Canadá y Chile: Una mirada desde las políticas públicas. Sin pie de imprenta. Recuperado de http://hivhealthclearinghouse.unesco.org/library/ documents/la-educacion-de-la-salud-sexual-de-los-adolescentes-en-canada-ychile-una-mirada.

Morgade, G., y Alonso, G. (2008). Cuerpos y sexualidades en la escuela. De la "normalidad" a la "disidencia". Buenos Aires, Argentina: Paidós.

OJeda SÁncheZ, A. (2010). ¿Alguien sabe quiénes somos? Mapas conceptuales sobre la diversidad sexual. En R. Parrini (2010). Instrucciones para vivir en un mundo diverso. Sujeto, cultura y diversidad sexual. Distrito Federal, México: Universidad Nacional Autónoma de México, Programa Universitario de Estudios de Género, Fondo de Desarrollo de las Naciones Unidas para la Mujer.

Osses Bustingorry, A.; Sánchez Tapia, I., y Ibánez Mancilla, F. M. (2006). Investigación cualitativa en educación. Hacia la generación de teoría a través del proceso analítico. Estudios Pedagógicos, 32(1): 119-133. Recuperado de http:// www.scielo.cl/scielo.php?script=sci_arttext\&pid=S0718-07052006000100007.

PARRINI, R. (2010). Instrucciones para vivir en un mundo diverso. Sujeto, cultura y diversidad sexual. Distrito Federal, México: Universidad Nacional Autónoma de México, Programa Universitario de Estudios de Género, Fondo de Desarrollo de las Naciones Unidas para la Mujer.

Reisigl, M., y Wodak, R. (2008). The Discourse-Historical Approach (DHA). Recuperado de http://www.researchgate.net/publication/251636976_The_ Discourse-Historical_Approach_(DHA). 
Richardson, D. (2000) Theory, Culture and Society. Rethinking Sexuality. Londres, Reino Unido: Sage Publications.

Ross, E., y Rapp, R. (1997). Sex and Society. A Research Note from Social History and Anthropology. En R. Lancaster y M. Di Leonardo (eds.) (1997). The Gender/ Sexuality Reader. Culture, History, Political Economy. Nueva York, Estados Unidos: Routledge.

SAYAGo, S. (2014). El análisis del discurso como técnica de investigación cualitativa y cuantitativa en las ciencias sociales. Cinta Moebio, 49: 1-10. DOI: 10.4067/S0717$554 X 2014000100001$.

Semanario Judicial de la Nación (2015). Tesis jurisprudencial 46/2015 10(a). En Primera Sala. Tesis jurisprudenciales 2015. Recuperado de https://www.scjn.gob.mx/ Primera_Sala/Tesis_Jurisprudenciales/TESIS\%20JURISPRUDENCIALES\%20 2015_PRIMERA\%20SALA.pdf.

Secretaría De Educación Pública (2011). Acuerdo 592 en el que se establece la articulación de la Educación Básica. Distrito Federal, México: Secretaría de Educación Pública. Secretaría de Educación Pública (2013). Programas de estudio 2011. Guía del maestro. Educación Básica. Secundaria. Ciencias. Distrito Federal, México: Secretaría de Educación Pública.

Secretaría de Educación Pública (2015). Principales cifras del sistema educativo mexicano 2014-2015. Distrito Federal, México: Dirección General de Planeación y Estadística Educativa, Secretaría de Educación Pública. Recuperado de http://planeacion.sep.gob.mx/assets/images/principales_cifras/2014_2015_ PRINCIPALES_CIFRAS_BOLSILLO.pdf.

Theumer, E., y García Muñoz, M. R. (2011). Cuerpos escolarizados: Pensar los embates de la integración desde la educación genérico-sexual. Artículo presentado en el I Congreso Internacional de la Red de Integración Latinoamericana 2011. Semana de la Integración, Universidad Nacional del Litoral. Santa Fe, Argentina, 9 al 12 de mayo de 2011. Recuperado de http://www.uncu.edu.ar/relacionesinternacionales/ upload/redilaeje15.pdf.

Van Dijk, T. A. (2004). Conferencia: Discurso y dominación. Bogotá, Colombia: Universidad Nacional de Colombia. Recuperado de www.bajofuego.org.ar/textos/ Discurso_y_dominacion.pdf.

VAn Dijk, T. A. (2009).Discurso y poder. Contribuciones a los estudios criticos del discurso, Barcelona, España: Gedisa. 
Wodak, R., y Meyer M. (2001). Methods of Critical Discourse Analysis. Londres, Reino Unido: Sage Publications.

YIn, R. K. (1984). Case Study Research. Design and Methods. California, Estados Unidos: Sage Publications. 